\title{
GUBERNAMENTALIDAD, DESPATOLOGIZACIÓN Y (DES)MEDICALIZACIÓN. INTERROGANTES SOBRE LA LEY DE IDENTIDAD DE GÉNERO ARGENTINA (2011-2014) GOVERNMENTALITY, DEPATHOLOGIZATION AND (DE) MEDICALIZATION. QUESTIONS ON THE LAW OF GENDER IDENTITY ARGENTINA (2011-2014)
}

Anahí Farji Neer ${ }^{1}$ Ana Mines ${ }^{2}$

RESUMEN: El presente artículo aborda los alcances de la despatologización y la desmedicalización en relación a las posibilidades simbólicas e institucionales abiertas por la Ley de Identidad de Género argentina (Ley 26.743/12). Focalizando en las tensiones entre medicalización-desmedicalización y patologización-despatologización analiza el debate parlamentario que llevó a la sanción de la Ley de Identidad de Género argentina. Prestando atención a ciertos procesos globales de mutación en las tecnologías de gobierno de los cuerpos y las subjetividades, indaga en los alcances y efectos de la medicalización de la identidad de género cuando, a partir de una legislación de alcance nacional, esta deja de ser entendida como enfermedad y se garantiza formalmente el acceso universal y gratuito a las tecnologías médicas de transformación corporal sustentándose en el derecho humano a la identidad de género. A través del análisis de contenido cualitativo de las transcripciones taquigráficas interpretamos los debates parlamentarios desarrollados durante los años 2011 y 2012 en las comisiones parlamentarias y en el recinto de las cámaras de Diputados y Senadores. En dicho marco se rastrean significados y tensiones en torno a la definición de "lo medicalizable", "lo patológico", "lo natural en relación al sexo" y "lo normal".

Palabras clave: despatologización; medicalización; identidad de género; derecho; ciudadanía.

\footnotetext{
${ }^{1}$ Magíster en Investigación en Ciencias Sociales y Doctoranda en Ciencias Sociales de la Universidad de Buenos Aires - UBA. Becaria del Consejo Nacional de Investigaciones Científicas y Técnicas - CONICET. Buenos Aires, D.F., Argentina. E-mail: anahifarji@hotmail.com

${ }^{2}$ Doctoranda en Ciencias Sociales de la Universidad de Buenos Aires - UBA. Becaria del Consejo Nacional de Investigaciones Científicas y Técnicas - CONICET. Buenos Aires, D.F., Argentina. E-mail: anamines@yahoo.com.ar
} 
ABSTRACT: This article focuses on tensions related to pathologization and depathologization as well as to medicalization and demedicalization brought up by recent Argentina's Gender Identity Law (Law No. 26.743/12). In May 2012, the Argentine National Congress approved the Gender Identity Law which regulates the change of name and sex of people whose gender expression does not agree with the one legally registered. Moreover, the law includes in the public health program the provision of hormone treatment and surgical intervention. In this article we inquire into the political and institutional discourses that circulated during parliamentary discussion of the Gender Identity Law during 2011 and 2012. Focusing on recent global mutations in contemporary biopolitics and in bodies and subject's technologies of government, this article explores the scope and effects of medicalization of gender identity. Hence, it inquires about the different discourses that lead to the approval of this national law regarding the fact that it disposes the free and universal access to medical technologies bodily transformation and that it is supported by the notion of human right to gender identity. Verbatim transcriptions of the debates developed in the House of Representatives and Senate are analyzed using the qualitative content analysis technique. Within this perspective, the meanings towards different notions of medicalization, pathologization, natural and normal are addressed.

Key words: pathologization; medicalization; gender identity; rights; citizenship.

\section{INTRODUCCIÓN}

En este artículo ${ }^{3}$ nos preguntamos por los alcances de la despatologización y la desmedicalización en relación a las posibilidades simbólicas e institucionales que abre la Ley de Identidad de Género argentina (Ley 26.743/12). La misma permite que el cambio registral de sexo y nombre sea realizado a través de un trámite administra-

\footnotetext{
${ }^{3}$ El presente artículo contiene avances parciales de las Tesis de Doctorado en curso de las autoras desarrolladas en el marco del programa de Doctorado en Ciencias Sociales de la Universidad de Buenos Aires. Una versión preliminar del mismo fue presentada en la IX International Conference de la International Association for the Study of Sexuality, Culture and Society (IASSCS) desarrollada en Buenos Aires durante el mes de Agosto de 2013.
} 
tivo sin requerir ningún tipo de autorización previa, ya sea de un equipo de expertos o pronunciamiento judicial. Asimismo, establece que la adecuación corporal al género autopercibido pueda realizarse -en caso de que las personas lo soliciten- a través de procedimientos médicos tales como intervenciones quirúrgicas "parciales o totales y/o tratamientos integrales hormonales" (Artículo 11 de la Ley 26.743/12). La Ley incluye tales tratamientos en el Plan Médico Obligatorio y de este modo establece su gratuidad en hospitales públicos y su cobertura por obras sociales y prepagas. A partir de su sanción el Estado Nacional reconoce a las identidades trans sin imponer para ello mediación judicial o médica.

Abordamos el debate parlamentario que llevó a la sanción de la Ley de Identidad de Género argentina focalizando en las tensiones entre medicalización-desmedicalización y patologización-despatologización. Tomando como marco las transformaciones de los últimos treinta años del campo médico -principalmente nacional, pero también pensando en cambios más generales de alcance global-, reflexionamos sobre los procesos de creciente y progresiva medicalización de las sociedades contemporáneas. Abordamos los mecanismos a través de los cuales el campo biomédico ${ }^{4}$ ha cifrado -y aún continúa cifrando- como enfermedades, anormalidades o trastornos toda una serie de episodios vitales que forman parte de los comportamientos de la vida cotidiana. Destacamos que, históricamente, la vivencia del género a contrapelo de las normas de género binarias ha sido incluida en dicha lógica medicalizadora a través de categorías y protocolos patologizantes, lógica que -a priori- parece ser desafiada por la Ley de Identidad de Género argentina.

Prestando atención a ciertos procesos globales de mutación en las tecnologías de gobierno de los cuerpos y las subjetividades (Rose, 2012) resulta de interés indagar en los alcances y efectos de

\footnotetext{
${ }^{4}$ Siguiendo a Eduardo Menéndez (2009), la noción de biomedicina responde a un tipo de saber específico, histórico y a la vez dinámico sobre la salud, el cuidado y la atención médica. A través de esta definición, Menéndez reconoce la existencia de "toda una variedad de saberes y formas de atención a los padecimientos (...) así como variados tratamientos e incluso diferentes tipos de curación" (2009: 25). La biomedicina, en tanto tipo particular de saber, se caracteriza por su identificación con la racionalidad científica, la ciencia positiva y su método. El saber biomédico se presenta como el más verdadero y eficaz generando una relación de subalternidad respecto a otros tipos de saberes sobre la salud, los cuerpos y los procesos de cuidado y atención.
} 
la medicalización de la identidad de género cuando, a partir de una legislación de alcance nacional, esta deja de ser entendida como enfermedad y se garantiza formalmente el acceso universal y gratuito a las tecnologías médicas de transformación corporal sustentándose en el derecho humano a la identidad de género. En este sentido, nos preguntamos ¿cómo se manifiesta allí la tensión medicalización-desmedicalización y patologización-despatologización?

Para responder este interrogante abordamos los debates parlamentarios que circularon con ocasión del tratamiento y sanción de la mencionada Ley. A través del análisis de contenido cualitativo (Andréu Abela, 1998) de las transcripciones taquigráficas, interpretaremos los debates parlamentarios desarrollados durante los años 2011 y 2012 tanto en las comisiones en las que los proyectos de Ley fueron tratados como en el recinto de las cámaras de Diputados y Senadores. Se tomarán dichos discursos como cristalizaciones emergentes de procesos más globales y complejos. Se prestará especial atención a las disputas en torno a la definición de "lo medicalizable", "lo patológico", "lo natural en relación al sexo" y "lo normal".

Comenzaremos desarrollando algunos de los principales conceptos teóricos que utilizaremos en relación al desarrollo de la biomedicina como tecnología de gobierno de los sujetos, en tensión con el contexto de producción y circulación local. Posteriormente historizaremos la producción de diagnósticos y protocolos de intervención de los cuerpos y subjetividades trans a partir de mediados de la década de 1960. Luego abordaremos los procesos de regulación de dichos cuerpos y subjetividades en Argentina a partir del una revisión del recorrido de la jurisprudencia local para, finalmente, ensayar una interpretación de los discursos que circularon al interior del Congreso Nacional ante la sanción de la Ley en estudio.

\section{LA BIOMEDICINA COMO TECNOLOGÍA DE GOBIERNO DE LOS SUJETOS}

La actual conformación del sistema biomédico y sanitario es producto de una multiplicidad de dimensiones. Hacer un breve 
repaso por la historia de la medicalización nos permite, como dice Eduardo Menéndez (2009), abordarla en tanto proceso social e histórico, susceptible de ser estudiado. Es decir que, a través de la historia de la biomedicina podemos, por ejemplo, indagar en su capacidad para construir síndromes que dan lugar a la elaboración de criterios, diagnósticos y de historias naturales de enfermedades 5 .

Durante la conformación de los estados nacionales en América Latina, proceso permeado por una lógica colonial, la expansión de la medicalización, fuertemente ligada al ordenamiento político de los individuos, la sociedad y el espacio. Estas funciones se entrecruzaron en el sistema sanitario que incluyó también un ordenamiento sexual y racial (Salessi 1995; Figari, 2009). A continuación destacaremos algunas características particulares vinculadas al desarrollo de la biomedicina y del sistema de salud argentino.

Las formas y modos que adquirió el sistema de salud local desde sus inicios ligados a la formación del estado nacional hasta la actualidad han estado necesariamente relacionados con los procesos económicos, sociales y políticos del país, la región y el mundo occidental. En su estudio La atención médica en la Argentina del siglo $X X$, Susana Belmartino (2005) propone analizar la segunda mitad del siglo en dos momentos. Por un lado, desde los años ' 40 hasta mediados de los '70 y por otro, durante los últimos 25 años del mismo siglo continuando hasta la actualidad. En relación al primero afirma que este período, signado por constantes interrupciones autoritarias, se caracteriza por la débil e inestable capacidad del Estado, en disputa con diferentes corporaciones, para "establecer reglas y sostenerlas a través de sanciones a su incumplimiento" (2005, p.155) ${ }^{6}$. En relación al segundo, afirma que el mismo se caracteriza por una fuerte penetración de las agendas y metodologías de las agencias

\footnotetext{
${ }^{5}$ En palabras del autor, "determinadas enfermedades han sido construidas por el propio saber médico, o por lo menos se acentuaron rasgos de las mismas, para justificar intervenciones por razones económicas, de control profesional o de control político" (2009, p.48).

${ }^{6}$ Esta etapa "es el momento de vigencia de determinadas instituciones y reglas de juego: el reconocimiento de la necesaria intervención del Estado destinada a garantizar la permanencia de un derecho socialmente reconocido a la salud y el consiguiente acceso a la atención médica; la efectiva intervención de sus agencias en las funciones de financiamiento, regulación y provisión del sistema de servicios; la defensa de un mecanismo solidario en la cobertura médica de la población; el predominio de la profesión médica en la delimitación de los aspectos técnicos de los servicios" (Belmartino, 2005, p.191).
} 
internacionales (principalmente OMS y $\mathrm{OPS}^{7}$ ) en materia de salud provocando efectos disímiles (Belmartino, 2005). Asimismo, destaca que los años '90 en adelante se caracterizan por un fuerte sesgo neoliberal que se cristalizó en la desregulación, liberalización y privatización de programas y servicios como también en el aumento de la desigualdad relativa en el acceso a los servicios hasta convertirse en fuente de exclusión y grave deterioro de la calidad de vida de sectores de menores recursos.

Siguiendo los desarrollos de Belmartino (2005) podemos afirmar que la historia argentina a partir de la segunda mitad del siglo XX está atravesada por una disputa entre dos modelos de estados: uno de compromiso y otro de Bienestar (Belmartino, 2005). La diferencia principal entre ellos radicaría en su capacidad de proponer y sostener reglas y regulaciones frente a las corporaciones privadas que tienen injerencia en los asuntos médicos.

Los procesos recién comentados permiten asumir que entre el Estado y las restantes instituciones que conforman el campo biomédico operan límites fluidos y porosos, así como dinámicas de demarcación mutua. La perspectiva de la gubernamentalidad desarrollada por Michel Foucault en su curso Seguridad, territorio, población (2006) son de gran utilidad para analizar dicha distinción y entrecruzamiento ${ }^{8}$.

Sería simplista entender al Estado como un actor homogéneo, productor de prácticas y discursos coherentes y sistemáticos. Por ello, distinguiremos las lógicas propias y específicas, rituales y capitales en disputa de cada uno de los campos que componen al aparato estatal. Empero, no por ello dejaremos de reconocer y trazar continuidades en las prácticas estatales en términos de estrategias o direccionalidades políticas. La idea de gobierno desarrollada por Foucault (2006) remite al "dominio que se puede ejercer sobre uno mismo y sobre los otros, y sobre el cuerpo, pero también sobre el alma y la manera de obrar" (2006: 149). El autor expresa que los Estados modernos se originan mediante la progresiva concentración

\footnotetext{
7 “Organización Mundial de la Salud” y "Organización Panamericana de la Salud” respectivamente. ${ }^{8}$ Foucault (2006) argumentó la necesidad de resituar al Estado moderno y sus prácticas dentro de una tecnología más general de poder. El autor allí sostenía que lo característico de la modernidad no sería tanto la estatización de la sociedad sino la progresiva gubernamentalización del Estado dando lugar a un novedoso modo de regulación, basado en la vida, al que llamó Biopoder.
} 
estatal de las artes de gobierno, colonizando las prácticas de gobierno preexistentes (de la casa, de las almas, de los niños, etc.). Las nociones de verdad, examen y obediencia son intrínsecas al esquema de la gubernamentalidad.

Proponemos pensar la relación entre el campo médico y el Estado como un vínculo de doble vía. Por un lado, las instituciones estatales en su afán por gobernar en base a preceptos verdaderos han recurrido -y continúan recurriendo- al discurso biomédico como discurso que produce y certifica la veracidad de los procesos de la vida a través del estudio científico de la naturaleza del cuerpo humano. Dentro de este esquema es posible entender a la biomedicina como institución encargada de ejercer el poder de policía de los cuerpos, garantizando su normalidad anatómica, funcional, erótica, etc. La idea de una naturaleza de los cuerpos que debe ser respetada -o bien, producida- ha sido el recurso fundamental para establecer normativamente lo que los cuerpos deben ser, frente a lo que de hecho son. Por otro lado, el campo estatal regula, limita y condiciona parcialmente a las instituciones que componen el campo biomédico, el cual goza de cierta autonomía relativa ${ }^{9}$.

\section{TENSIONES ENTRE LA MEDICALIZACIÓN Y LA DES- MEDICALIZACIÓN ¿PROCESOS ANTAGÓNICOS O CON- TINUOS?}

La noción de medicalización resulta útil a fin de ubicar la Ley de estudio en una trama de procesos sociales complejos, dado que la misma permite abordar críticamente los efectos disímiles que lleva consigo la injerencia del sistema de salud en los procesos individuales, subjetivos y comunitarios ${ }^{10}$. Según Rose "la biomedicina cambia qué

\footnotetext{
${ }^{9}$ Susana Belmartino (1999) afirma que la profesión médica dispone de una "dosis significativa de autonomía" (1999, p.20) relacionada no sólo al proceso de delegación de autoridad por parte del Estado sino también al aumento de una creciente profesionalización de la sociedad. Al mismo tiempo, reconoce matices en este proceso según las diferentes regiones del país evidenciando la relación que existe entre este proceso y la conformación de elites y corporaciones.

${ }^{10}$ Como dice Graciela Natella, "la fuerte tendencia a medicalizar los problemas de salud y asumir que su solución primera involucra el tratamiento médico, genera que los hacedores de políticas, en el mejor de los casos, pongan el foco en aumentar el acceso financiero y geográfico a los servicios de salud para las poblaciones vulnerables, descuidando en oportunidades causas sociales y económicas de la vulnerabilidad y disparidad en salud" (2010, p.46).
} 
es un ser humano" (2012, p. 49). En este sentido, cabe afirmar que el desarrollo de los últimos años de técnicas y tecnologías biomédicas no sólo han provocado mutaciones en las relaciones financieras e institucionales ligadas a estos desarrollos, sino también en las ideas que tenemos en torno a la vida, lo vivible, al cuerpo, las terapias, etc.

Entendemos por medicalización un proceso que reduce la complejidad de los problemas vitales a cuestiones de orden médico o psicológico, centralizando en la persona la causa y tratamiento del malestar, desestimando los determinantes sociales de la salud y desactivando las potencias individuales y colectivas. Menéndez (2009) sostiene que la medicalización implica cifrar en términos de salud y enfermedad -haciendo foco en la enfermedad y en la terapéuticatoda una serie de episodios vitales que son parte de los comportamientos de la vida cotidiana de los sujetos.

"Si bien casi todas las formas y saberes se preocupan por la salud e incluso la biomedicina habla de producir salud, la salud positiva o de estilos de vidas saludables, lo cierto es que casi la totalidad de actividades de los diversos saberes y formas de atención actúan básicamente respecto de los padecimientos y enfermedades y no respecto de la producción de salud. Ello es así no sólo porque lo proponen e impulsan los curadores, sino porque además lo solicitan los sujetos y grupos sociales, ya que demandan básicamente acciones sobre sus padeceres más que sobre su salud" (Menéndez, 2009, p.28).

Entonces, resulta interesante indagar en las dimensiones que conforman la perspectiva biomédica para representar el cuerpo y sus procesos.

Por otro lado, siguiendo el análisis de Nikolas Rose (2012), el desarrollo de la biomedicina responde a una lógica de optimización de los cuerpos y ya no principalmente a la dicotomía salud/enfermedad. En palabras del autor, "las tecnologías contemporáneas no buscan meramente curar la enfermedad una vez que se manifiesta, sino controlar los procesos vitales del cuerpo y la mente: sugiero que son tecnologías de optimización" (2012, p.48). 
Siguiendo a Peter Conrad (1992), la medicalización construye los problemas sanitarios como asuntos individuales, extiende la esfera del control médico sobre el comportamiento humano y otorga autoridad epistémica al campo médico sobre hechos que generalmente escapan a su plena comprensión. Señala que en la actualidad se trata de un proceso integrado por distintos actores, cada uno con su rol y sus intereses: la comunidad médica, los pacientes -aislados u organizados- y la corporación farmacéutica. Conrad, en su estudio ya clásico sobre la medicalización de la desviación (1982), dio cuenta del modo en que prácticas entendidas como inmorales, pecaminosas o criminales con el despegue del sistema médico pasaron a tener estatus y significado médico en tanto patologías que debían ser tratadas de determinada manera. Ello le otorgó a la medicina su carácter moral. No obstante, destacó que la medicalización es un proceso que excede y rebasa al mero control social de la desviación. En aparente antagonismo al proceso de medicalización nos encontramos frente a procesos de desmedicalización. Conrad (1992) define como desmedicalización el trayecto histórico mediante el cual un asunto deja de poseer una definición médica y los tratamientos o las tecnologías médicas dejan de plantearse como solución.

Pero el proceso de medicalización-desmedicalización no es un proceso lineal o progresivo que lleva necesariamente a la emancipación de los cuerpos y los sujetos, reviste importante complejidad e incluso cierta ambivalencia. Tomando como caso paradigmático la medicalización de la homosexualidad, Conrad (1982) destaca que su conceptualización como patología a fines del siglo XIX significó la protección de los sujetos contra la represión legal, aunque implicó su ingreso en los violentos mecanismos de las terapias correctivas. Hacia la década de 1960 la conformación de grupos que hicieron de la despatologización una consigna de lucha en un marco de crítica generalizada a la disciplina psiquiátrica generó las condiciones para que en 1973 la APA $^{6}$ decidiera incluir la homosexualidad como enfermedad del DSM III sólo cuando la misma estuviera acompañada de malestar o sufrimiento psíquico. La desmedicalización de la homosexualidad fue solo parcial, dado que con la emergencia del virus 
del SIDA, la homosexualidad volvió a caer en las redes de la medicalización. Así la homosexualidad (la masculina particularmente) fue designada como grupo de riesgo y sus formas de vida fueron nuevamente ubicadas bajo la lupa médica en un marco de reforzamiento del control sobre las prácticas sexuales (Conrad, 2007).

Cabe sostener entonces que medicalización y desmedicalización deben ser entendidos como tácticas específicas dentro de una estrategia global de gobierno de los cuerpos, y que, en relación a las particularidades de cada contexto específico se mantiene en permanente mutación y reconfiguración.

\section{NOTAS EN TORNO A LA HISTORIA DE LA MEDICALI- ZACIÓN Y REGULACIÓN ESTATAL LOCAL DEL TRAVES- TISMO Y LA TRANSEXUALIDAD}

El campo médico tomó a su cargo el gobierno de las formas de vida que desafían el binarismo genérico cifrándolas e instaurándolas socialmente como enfermedades, particularmente, como patologías mentales. Para ello se valió de las categorías diagnósticas del travestismo y la transexualidad. El nacimiento de la primera fue fruto de los desarrollos de la sexología clínica norteamericana y europea de principios del siglo XX, mientras que la segunda nació a mediados de la década de 1960 de la mano del despegue del conocimiento endócrino y la técnica quirúrgica ${ }^{11}$ en Estados Unidos.

Fue esta última categoría diagnóstica la que tuvo mayor aceptación y circulación al interior del campo médico. Su creador, el endocrinólogo alemán Harry Benjamin estableció las bases para diagnosticar el verdadero transexualismo en su libro El fenómeno transexual publicado en el año 1966. Estos desarrollos fueron profundizados en los Standards of Care for Gender Identity Disorders (SOC) editados en el año 1979.

Benjamin estableció un método estandarizado para determinar el diagnóstico de la transexualidad verdadera y así diferenciarla

\footnotetext{
${ }^{11}$ Siguiendo a Berenice Bento (2006), el término transexual fue acuñado por primera vez por Magnus Hirschfeld en el año 1910. El mismo utilizó el concepto de "transexual psíquico" para referirse a lo que entendía como una modalidad particular de travestismo fetichista. El término volvió a ser utilizado recién en el año 1949 por parte de David O. Caldwell, psiquiatra estadounidense.
} 
de la psicosis. En el caso de las identidades trans, el proceso de medicalización estuvo inexorablemente ligado a la patologización. De hecho, podríamos decir que fue consecuencia de la patologización: las formas de vivir el género y la corporalidad a contramano de las normas binarias de género fueron concebidas como patologías cuya única terapéutica posible fue la medicalización de sus cuerpos, mediante tratamientos hormonales y quirúrgicos.

Los manuales de diagnóstico de uso mundial incorporaron la categoría diagnóstica en las mismas ediciones en las que la homosexualidad era eliminada (el CIE ${ }^{12}$ en su $9^{\circ}$ versión de 1978 y el DSM ${ }^{13}$ III de 1980), lo cual implicó una repatologización de la disidencia sexo genérica. En las sucesivas versiones de los manuales, el mismo fue reemplazado por el de "Trastorno de la Identidad de Género" con el fin especificar su vinculación con la dimensión de género y diferenciarlo más claramente de la orientación sexual. Como respuesta a la fijación e institucionalización de dichos diagnósticos patologizantes, desde el año 2007 en Barcelona se organizó la Campaña "Stop Trans Patologization"14. Originalmente convocada por grupos activistas españoles y luego extendida y replicada a nivel mundial, la misma busca retirar la categoría de "disforia de género" y "trastornos de la identidad de género" del DSM y la CIE, proponiendo la inclusión de una mención no patologizante. En el DSM 5 en su versión del año 2013 reemplazó dicha categoría por la de "Disforia de género". De este modo, solo es entendido como trastorno cuando lo acompaña alguna forma de sufrimiento psíquico, tal como fue reconceptualizado en la versión de los SOC del año 2011.

En el contexto local, el diagnóstico y sus protocolos asociados circularon no sólo al interior del campo médico sino también en el ámbito jurídico. A mediados de la década del '90 se dio la incorporación de categorías por parte del campo judicial local. La entonces vigente Ley de "Ejercicio de la Medicina"15 prohibía cualquier intervención quirúrgica que alterara la genitalidad de las personas

\footnotetext{
${ }^{12}$ Clasificación Internacional de Enfermedades editado por la Organización Mundial de la Salud. ${ }^{13}$ Manual Diagnóstico y Estadístico de los Trastornos Mentales, editado por la American Psychiatric Association, (APA).

${ }^{14} \mathrm{http}: / /$ stp2012.info/old/es

${ }^{15}$ Ley $\mathrm{N}^{\circ} 17.132 / 67$
} 
y la "Ley del Nombre"16 establecía que para modificar el nombre asentado en la partida de nacimiento debía probarse frente a un juez la existencia de un "justo motivo". Por ello quienes quisieran intervenir su cuerpo como quienes quisieran modificar su nombre y sexo en el DNI debían solicitar autorización judicial. Con anterioridad al año 1997, los pedidos fueron negados. Los jueces consideraban que se trataba de demandas originadas en una desviación psicológica y que el campo judicial no podía tomar decisiones que desafiaran las leyes naturales (Farji Neer, 2013). Los pedidos que comenzaron a ser autorizados a partir de dicho año adoptaron la perspectiva, retórica y filosofía de los protocolos vigentes en el campo médico. A través de las pericias médico-psiquiátricas los jueces debían asegurarse que el pedido obedecía a un cuadro de transexualismo verdadero, tal como lo estipulaba el diagnóstico. La exposición del sufrimiento era parte del ritual jurídico necesario para acceder al cambio de nombre y sexo registral así como a la realización de intervenciones de transformación corporal. De este modo, la autorización de los pedidos oficiaba como una suerte de reparación a víctimas de una patología particular. Dado que la estructura de los protocolos incluía obligatoriamente la realización de la cirugía de reasignación sexual como última etapa del tratamiento, los pedidos de cambio de nombre y sexo registral sólo se autorizaban si dicha cirugía hubiera sido realizada previamente (generalmente en el extranjero) y hubiera sido corroborada mediante pericias médicas o si, en su defecto, quienes pedían el cambio registral se comprometían expresamente a realizarla (Cabral, 2003; 2008; Farji Neer, 2013).

A finales del año 2010 se emitió el primer fallo judicial que autorizaba el cambio de nombre y sexo legal eludiendo el requisito del diagnóstico y las pericias médicas. Allí se ponderó la identidad autopercibida por sobre la corporalidad y tuvieron valor de pruebas jurídicas las declaraciones de los/as testigos presentados/as por la/el solicitante. Asimismo, se amparaba en el discurso de los derechos humanos y en el respeto por la autonomía de decisión de las personas. Bajo los mismos principios, comenzaron a ser emitidos una serie de

${ }^{16}$ Ley $\mathrm{N}^{\circ} 18.248 / 69$ 
fallos con similar resolución (Farji Neer, 2013). Dichos fallos sirvieron de incentivo para que los proyectos de Ley de Identidad de Género que venían siendo presentados desde el año 1997- pudieran ingresar en la agenda parlamentaria. La sanción de una Ley de Identidad de Género se había ubicado como la demanda central de las organizaciones de travestis, transexuales y transgénero argentinas de la última década.

Habiendo analizado cómo los procesos de medicalización y patologización constituyeron engranajes claves en el desarrollo de la gubernamentalidad contemporánea nos preguntamos en qué medida puede una regulación estatal despatologizar y desmedicalizar los cuerpos. A fin de ensayar una respuesta a este interrogante analizaremos las discusiones parlamentarias que llevaron a la sanción de la Ley de Identidad de Género haciendo foco en cómo operaron las nociones de "lo medicalizable", "lo patológico", "lo natural en relación al sexo" y "lo normal".

\section{LA LEY: UN MOMENTO ACOTADO EN LA HISTORIA DEL VÍNCULO ENTRE LAS ORGANIZACIONES DE TRAVES- TIS, TRANSEXUALES Y TRANSGÉNERO Y EL ESTADO}

\subsection{Antecedentes}

Las organizaciones de travestis, transexuales y transgénero de Argentina surgieron a comienzos de la década del ' $90^{17}$. Con anterioridad a su conformación, distintas activistas travestis venían acompañando las reivindicaciones de aquellos colectivos conformados durante la reapertura democrática cuyas demandas se centraban en la identidad homosexual. A comienzos de los ' 90 la comunidad travesti, transexual y transgénero -nucleada bajo la identidad travesti, luego ampliada a las categorías de transexual y/o transgénero- co-

\footnotetext{
${ }^{17}$ En 1991 se creó Transexuales por el Derechos a la Vida y la Identidad (Transdevi) y Asociación de Travestis Argentinas (ATA). Luego Travestis Unidas (TU), Organización de Travestis y Transexuales de la Argentina (OTTRA), Tratado del Derecho a la Identidad Personal (TDI) y la Asociación por la Identidad de las Travestis (ALIT), posteriormente Asociación de Lucha por la Identidad Travesti- Transexual (ALITT) (Fernández, 2004; Hiller, 2011). En el año 2002 se crea el Movimiento Antidiscriminatorio de Liberación (MAL) y en el 2007 Futuro Transgenérico. También se conformaron colectivos que politizan la transexualidad masculina, como es el caso del grupo Teatro de Operaciones y posteriormente la agrupación Hombres Trans Argentinos. A fines del año 2000 se crearon agrupaciones en distintas provincias del interior del país (Farji Neer, 2013).
} 
menzó a hacer públicas sus demandas en el ámbito de la Ciudad de Buenos Aires. La demanda central era la descriminalización de sus identidades y el cese de la persecución policia ${ }^{18}$. La primera política pública de la Ciudad de Buenos Aires obtenida a nivel ministerial se obtuvo en el año 2003 por parte de la entonces Secretaría de Educación. La Resolución $\mathrm{N}^{\circ} 122$ recomendaba el reconocimiento a la identidad autopercibida de travestis y transexuales en los ámbitos educativos de dicha jurisdicción ${ }^{19}$. Paralelamente, en articulación con el campo académico feminista, las mencionadas organizaciones elaboraron informes de la situación social de su comunidad (Berkins, 2007; Berkins y Fernández, 2005). Estos graficaban su extrema vulnerabilidad a nivel sanitario, habitacional, educacional y laboral.

La politóloga Renata Hiller (2011) destaca tres aspectos que caracterizaron a las organizaciones sexo-políticas durante los años 2000. Por un lado, la federalización de las organizaciones y reivindicaciones, tomando como caso paradigmático la conformación de la Federación Argentina de Lesbianas, Gays, Bisexuales y Trans (FALGB) en el año 2005. Por otro lado, señala una nueva modalidad de vinculación con los partidos políticos. Al respecto, la autora explica que a partir de la crisis del año 2001 varios/as militantes LGTB se acercaron a la política partidaria y paralelamente, algunos partidos políticos - de izquierda particularmente ${ }^{20}$ - comenzaron a incorporar a sus plataformas programáticas las demandas de dichas organizaciones. Por último, a partir del año 2003 la autora subraya la imbricación de las organizaciones LGBT con el Estado. Señala que con la gestión presidencial de Néstor Kirchner (Frente para la Victoria/ Partido Justicialista - FPV/PJ) a partir del año 2003, se afianzó una estrategia política centrada en la incorporación a la estructura del

\footnotetext{
${ }^{18}$ A partir de la derogación de los Edictos Policiales en el año 1996 y con la autonomización de la Ciudad de Buenos Aires, las organizaciones levantaron la bandera del reconocimiento a la identidad autopercibida en los ámbitos institucionales y el acceso efectivo a los derechos básicos (salud, educación, trabajo y vivienda).

${ }^{19}$ Como antecedente a dicha normativa cabe destacar el trabajo de prevención de VIH-Sida dirigido a la población travesti llevado a cabo por la Coordinación Sida de la Secretaría de Salud Gobierno de la Ciudad de Buenos entre los años 2002 y 2003 y el programa de "Construyendo Ciudadanía Travesti" de la Defensoría del Pueblo de la Ciudad de Buenos Aires durante la gestión de la Dra. Diana Maffía (1998-2003) (Raffo, 2006).

${ }^{20}$ Grupo LGBT del Partido Socialista, Secretaría de la Diversidad Sexual del Partido Socialista de la Ciudad de Buenos Aires, Jóvenes por la Igualdad (en el ARI-Coalición Cívica); Carne Clasista (en el Movimiento Al Socialismo, MAS).
} 
Estado de miembros de distintas organizaciones sociales. En el caso de la organizaciones sexo políticas, dichos cuadros fueron incorporados, mayormente, mediante la creación de los Foros de Organizaciones de la Sociedad Civil del INADI (Instituto Nacional contra la Xenofobia, la Discriminación y el Racismo) ${ }^{21}$ (Hiller, 2011).

En el marco del proceso de articulación del Estado con una variedad de actores e instituciones, la demanda de una Ley de Identidad de Género que regule el cambio de nombre y sexo legal en los DNI (Documento Nacional de Identidad) fue planteada por las organizaciones como paso necesario para lograr la plena inclusión social y el acceso a los derechos básicos para su comunidad. La presentación de distintos proyectos de Ley comenzó en el año 1997. Sin embargo, hasta el año 2011 ninguno había llegado a ser discutido por las comisiones parlamentarias.

El tratamiento y final sanción de la Ley debe situarse en un marco más amplio de reconfiguraciones en el vínculo entre el Estado y los colectivos sexo-políticos locales. En este sentido, la sanción de la Ley de Matrimonio Igualitario ${ }^{22}$ en el año 2010 marcó un punto de inflexión abriendo una ventana de oportunidades políticas a partir de la cual las organizaciones trans locales orientaron su trabajo en pos de una Ley de Identidad de Género.

$\mathrm{La} \mathrm{FALGBT}^{23}$ y el entonces recientemente conformado Frente Nacional por la Ley de Identidad de Género ${ }^{24}$, presentaron sus propios proyectos de Ley a través de dos diputadas del Frente Para

\footnotetext{
${ }^{21}$ El Instituto Nacional contra la Discriminación, la Xenofobia y el Racismo (INADI) es un organismo descentralizado que fue creado mediante la Ley $\mathrm{N}^{\circ} 24.515$ en el año 1995 y comenzó sus tareas en el año 1997. Desde el mes de marzo de 2005, por Decreto Presidencial No 184 , se ubicó en la órbita del Ministerio de Justicia, Seguridad y Derechos Humanos de la Nación. Los "Foros de la Sociedad Civil" son espacios propuestos por el INADI como ámbitos de articulación entre el Estado y organizaciones sociales a fin de elaborar conjuntamente propuestas de políticas públicas, seguimiento y monitoreo. Algunos de los foros propuestos por el Instituto son el foro de la juventud, migrantes y refugiados/as, discapacidad, afrodescendientes y diversidad sexual.

${ }^{22}$ Ley N ${ }^{\circ} 26.618$

${ }^{23}$ Federación de organizaciones LGBT conformada en el año 2005 por las organizaciones ATTTA (Asociación de Travestis Transgéneros y Transexuales de Argentina); La Fulana (organización de mujeres lesbianas y bisexuales de Buenos Aires); Nexo Asociación Civil (organización gay de Buenos Aires fundada en 1992); VOX Asociación Civil (organización LGBT de la provincia de Santa Fe fundada en 1998) y la Fundación Buenos Aires Sida (Hiller, 2011).

${ }^{24}$ Coalición de organizaciones creada en el año 2010 y conformada por Putos Peronistas, A.L.I.T.T., Cooperativa "Nadia Echazú", Hombres Trans Argentinos, Movimiento Antidiscriminatorio de Liberación (M.A.L.), Futuro Trans, Encuentro por la Diversidad (Córdoba), MISER, Antroposex, Viudas de Perlongher, Jóvenes por la Diversidad, Escénica Arte y Diversidad, Cero en Conducta (Santiago del Estero), ADISTAR-Salta, Comunidad Homosexual Argentina, Apid, Crisálida (Tucumán), Ave Fénix, AMMAR Córdoba y activistas independientes.
} 
la Victoria (Expediente $\mathrm{N}^{\circ}$ 7644-D-2010, 7643-D-2010 y Expediente $\mathrm{N}^{\circ}$ 8126-D-2010). Otros/as diputados/as también elevaron sus proyectos. Estos fueron presentados por representantes del Partido Socialista (Expediente $\left.N^{\circ} 1879-\mathrm{D}-2011\right)$ y de la Unión Cívica Radical (Expediente $\mathrm{N}^{\circ}$ 7243-D-2010). El proyecto del Frente Nacional por una Ley de Identidad de Género unificaba la regulación del régimen de cambio registral de sexo con la de los tratamientos hormonales e intervenciones sobre el cuerpo, incluyéndolos en el Plan Médico Obligatorio y determinando su gratuidad. Ambos aspectos fueron incorporados en la Ley aprobada. La Federación presentó dos proyectos diferenciados, uno orientado a regular el cambio registral y otro a regular el aspecto sanitario.

El 18 de Agosto del 2011 se inició el tratamiento de los cuatro proyectos en las Comisiones de Legislación General y Justicia de la Cámara de Diputados. En dicha oportunidad fueron convocados al debate, referentes de las organizaciones de travestis, transexuales y transgénero locales $^{25}$, un jurista, el entonces interventor del INADI y un Juez del Fuero en lo Contencioso Administrativo y Tributario de la Ciudad $\mathrm{Au}-$ tónoma de Buenos Aires ${ }^{26}$. El 30 de Noviembre, en la última sesión del año, aquel dictamen fue tratado en la sesión de la Cámara de Diputados, obteniendo 167 votos a favor, 17 en contra y 6 abstenciones.

El 17 de Abril del año 2012 al reiniciarse el trabajo parlamentario el proyecto ya aprobado en la Cámara de Diputados fue tratado conjuntamente por las comisiones de Legislación General y Población y Desarrollo Humano de la Cámara de Senadores. Allí fueron convocados referentes de las organizaciones trans y sexo políticas ${ }^{27}$, y del Ministerio de Trabajo ${ }^{28}$. El 9 de mayo del 2012 tuvo su sanción

\footnotetext{
${ }^{25}$ Marcela Romero y Claudia Pia Baudracco integrantes de ATTTA y representantes de la Federación LGBT; Mauro Cabral, codirector de Acción Global por la Igualdad Trans y miembro del Frente Nacional por una Ley de Identidad de Género; Alba Rueda de la Asociación Civil 100\% Diversidad y Lohana Berkins integrante de ALITT y del Frente Nacional de Ley de Identidad de Género. ${ }^{26}$ Doctor Andrés Gil Domínguez, Pedro Mouratián, y Doctor Guillermo Scheibler, respectivamente. ${ }^{27}$ Martín Canevaro miembro de la agrupación 100\% Diversidad y Derechos, quien habló en nombre del INADI, Mauro Cabral (GATE), Diana Sacayán (MAL), Lohana Berkins (ALITT), Marlene Wayar (Futuro Transgenérico), Blas Radi y un/a integrante de la Agrupación Nacional de Putos Peronistas en representación del Frente Nacional por la Ley de Identidad de Género; Esteban Paulón y Marcela Romero (ATTTA) en representación de la Federación Argentina de Lesbianas, Gays, Bisexuales y Trans; Alba Rueda, representante de 100\% Diversidad y Derechos; César Cigliutti de la Comunidad Homosexual Argentina; Yanina Moreno.

${ }^{28}$ Sra. Matilde Cerruti.
} 
definitiva en la Cámara de Senadores con 55 votos a favor y una abstención, sin votos negativos.

\subsection{En el recinto}

\subsubsection{Lo normal}

Al adentrarnos en los discursos que circularon al debatirse la Ley, reconocemos que un factor necesario para su sanción radicó en el corrimiento del horizonte de lo "normal" en términos sexo-genéricos. Al hacerlo, las organizaciones trans le disputaron al Estado la ampliación de los límites de la ciudadanía -en el sentido arendtiano del derecho a tener derechos- y en esta misma contienda disputaron los límites de lo normal, es decir, de lo inteligible no sólo jurídicamente, sino también culturalmente.

Para que eso sea posible, las organizaciones recurrieron al lenguaje de los Derechos Humanos como estrategia que permitió traducir sus demandas a un lenguaje audible por el Estado, particularmente por el campo parlamentario. La relación entre el Estado y las organizaciones trans estuvo mediada por el recurso discursivo de la idea de reparación a las víctimas. En este sentido, una reunión de comisión, la activista Marcela Romero (ATTTA) expresó: "Siempre digo que somos las olvidadas de la democracia. Y no queremos ser más las olvidadas de la Democracia”.

En el recinto, el diputado Miguel Ángel Barrios (Partido Concertación Cívica por la Provincia de Santa Fé) expresó lo siguiente: "Si pudiéramos poner en un ranking de vulnerabilidad a los distintos colectivos que conforman al mundo de la diversidad, las personas travestis, transexuales y transgénero se ubican lamentablemente en el primer lugar". Asimismo, la Diputada Laura Alonso (Partido Alianza PRO - Propuesta Republicana por la Ciudad Autónoma de Buenos Aires) refirió:

"Hoy estamos dando visibilización a uno de los grupos del colectivo más discriminado de nuestra sociedad; me refiero a las personas trans, término con el cual aludiré genéricamente a los individuos 
travestis, transgénero, transexuales e intersex. Ellas han sido, quizás, las más castigadas, discriminadas, torturadas, asesinadas, condenadas injustamente a lo largo de la historia argentina tanto por las dictaduras como por las democracias (...) las personas trans son víctimas de crímenes, de odio, de situaciones de abuso por parte de la autoridad pública y de la pandemia del VIH sida”.

El recurso discursivo de la reparación a víctimas justificó que el Estado ponga sus fondos públicos a disposición de las necesidades de las personas trans y abrió una serie de tensiones en relación a los protocolos vigentes al interior del campo médico, dislocando sentidos en relación a lo patológico, lo medicalizable y lo natural en relación al sexo.

\subsubsection{Lo patológico}

Con respecto a la patologización, observamos una suerte de giro epistemológico por parte de quienes encarnaron los debates. Ello es así dado que la patologización fue entendida -por sí misma y como fundamento para la judicialización de los pedidos de cambio de nombre y sexo registral- como práctica violatoria de los derechos humanos de las personas trans. En palabras de la Diputada Vilma Ibarra (Partido Frente para la Victoria por la Ciudad Autónoma de Buenos Aires):

\footnotetext{
“(...) no exigimos procedimientos quirúrgicos, diagnósticos ni métodos patologizantes. Toda legislación moderna avanza en este sentido. No queremos una ley que nazca vieja. Nuestra propia ley de salud mental 26.657, (...), prohíbe hacer diagnósticos en el campo de la salud mental sobre la base de la identidad de género y de la identidad sexual. En el mismo sentido se expiden los principios de Yogyakarta y Human Rights Watch”.
}

El discurso médico-psiquiátrico dejó de ser cifrado como el discurso de verdad en base al cual las instituciones estatales deben regular. Por el contrario, fue entendido como una ficción discursiva que enmascara mecanismos sociales de discriminación y exclusión. 
En este sentido, la verdad en base a la cual el Estado debe legislar fue ubicada en el discurso de los sujetos. El paradigma internacional de los Derechos Humanos en relación a la despatologización de las identidades trans (los "Principios de Yogyakarta sobre la aplicación de la legislación internacional de derechos humanos en relación con la orientación sexual y la identidad de género" ${ }^{29}$ ) y las normativas locales en el campo de la salud mental permitieron avalar normativamente dicha posición ${ }^{30}$.

Si por un lado la patologización fue criticada, por otro, las prácticas médicas de transformación corporal mediante hormonización y cirugías fueron entendidas como prácticas que debían ser no sólo avaladas por el Estado -legalizadas- sino también democratizadas -financiadas por el sistema público de salud y cubiertas por los sistemas privados. Asimismo, se disponía la no obligatoriedad de sometimiento al dispositivo médico ni a sus técnicas. Ni la medicalización ni la judicialización fueron establecidas como condiciones para acceder al cambio registral de sexo y a los tratamientos hormonales e intervenciones quirúrgicas. Según el Diputado Juan Pedro Tunessi (Partido Alianza Acuero Cívico y Social por la Provincia de Buenos Aires):

"La gente tiene derecho a adecuar su cuerpo a la identidad que esa persona percibe o cree percibir. No hay derecho si no existe el mismo reconocimiento en las obras sociales. (...). Entonces, toda prestación está contemplada, porque queda incluida en el Plan Médico Obligatorio para hacer verdaderamente efectivo este derecho".

\subsubsection{Lo medicalizable}

Diferentes legisladores/as de ambos recintos manifestaron cierta incomodidad frente a exigir al sistema público de salud la cobertura en la atención de las demandas de personas trans, dejando entrever que desde su perspectiva, las prioridades sanitarias debieran ser dadas al tratamiento de patologías. En palabras del Diputado Julián Obligio (Partido Alianza PRO - Propuesta Republicana por

\footnotetext{
${ }^{29} \mathrm{http}$ //www.yogyakartaprinciples.org/

${ }^{30}$ Ley Nacional de Salud Mental No 26.657
} 
la Ciudad Autónoma de Buenos Aires): “(...) me parece que en la Argentina hay otros temas prioritarios para ser cubiertos con fondos públicos o por las obras sociales; hay ciertas patologías concretas que deberían ser cubiertas y que en este momento no lo son”.

La cuestión del financiamiento y las prioridades de financiación se plantearpn como aristas que generaron cierta polémica, trayendo nuevamente a cuento la tensión que se plantea entre los procesos de medicalización y (des)patologización. En palabras de la Senadora Graciela Di Perna (Partido Frente por la Integración por la Provincia de Chubut):

"Otro de los problemas en los que peca de omisión este proyecto es la financiación. Se dice que las personas que padecen este conflicto entre sexo y percepción de género no son enfermos, pero sin embargo se cargan sobre el sistema sanitario tratamientos quirúrgicos y hormonales con los mismos recursos" (Senadora Di Perna).

En el marco del debate se impuso el hecho de entender el acceso a las tecnologías de transformación corporal como parte intrínseca del derecho a la identidad, fundante del ingreso a la ciudadanía. Es decir que, según los dichos de algunos/as legisladores y legisladoras, no habría posibilidad de garantizar el ejercicio pleno de la ciudadanía si no se puede construir previamente una corporalidad acorde a propia identidad genérica. Según la Senadora Sonia Escudero (Alianza Partido Justicialista - Frente Justicialista para la Victoria - Provincia de Salta):

"Sé que hay senadores que tienen algunas objeciones a que se incorpore en el plan médico obligatorio el acceso a los tratamientos, a la intervención quirúrgica, a tratamientos hormonales, pero la verdad es que si las cifras nos muestran que el 95 por ciento de estas personas están fuera del acceso de los derechos, me parece que lo menos que podemos conceder es este derecho. Y digo esto, porque en términos económicos el impacto es ínfimo, pero en términos de acceso a los derechos, el impacto es enorme". 
De este modo, se expresó que la ciudadanía tiene cuerpo: un cuerpo generizado y sexualizado. La construcción de la corporalidad deseada se postularía así como pre-requisito para el ejercicio de los derechos económicos, sociales y políticos. En su exposición en la discusión del proyecto de Ley en el Senado, la activista Diana Sacayán (M.A.L.) ${ }^{31}$ lo expresó del siguiente modo:

"Es necesario que tomemos conciencia, en el contexto en el que hoy nos atrevemos a discutir, sobre la ley de identidad de género que habla también de acceso a la tecnología biomédica, a la salud pública. Con relación a esto, cabe señalar que muchas compañeras murieron por aplicación de silicona industrial, muertes que, por supuesto, puede ser absolutamente evitable si este Honorable Senado convierte en ley este proyecto que para nosotras es sumamente necesario para que parte de nuestra comunidad, como somos las personas travestis y transexuales, también podamos tener acceso a los derechos económicos, políticos y sociales como el resto de todos los ciudadanos y todas las ciudadanas".

En este mismo sentido, Mauro Cabral (investigador, historiador y miembro de la organización internacional Global Action for Trans Equality) en su exposición durante la discusión de los proyectos en la Cámara de Diputados expresó:

"Es necesario expandir ese compromiso hacia un econocimiento integral de la identidad de género. Y ese reconocimiento integral requiere algo más que el cambio registral, algo más que el reconocimiento de nuestro nombre y de nuestro sexo. Lo que requiere de manera indisociable es el reconocimiento de que la identidad de género se encarna en un cuerpo que cada cual, de manera autónoma, pueda sentir y reconocer como propio".

${ }^{31}$ Movimiento Antidiscriminatorio de Liberación: http://grupomal.blogspot.com.ar/ 


\subsubsection{Lo natural en relación al sexo}

En lo que refiere a los cambios de nombre y sexo legal en los registros identificatorios oficiales, se expresaron discusiones en las que se señaló el carácter simbólico de dichos registros en relación a la historia institucional del país. Según la Senadora Di Perna:

"En primer lugar, este proyecto propone la adulteración de un documento público, como es la partida de nacimiento. Sabemos que este no es el único documento que acredita el sexo y la identidad en un recién nacido. También lo hacen la historia clínica perinatal, la planilla identificatoria del recién nacido, la libreta sanitaria y los libros de parto que se llevan en maternidades y hospitales. Los datos de estos registros los aportan profesionales que tienen también una responsabilidad penal ante los mismos, y deben ser coincidentes con los datos de la partida de nacimiento. Debemos recordar que este tipo de registros han sido solicitados por la Justicia para esclarecer casos de niños apropiados ${ }^{32}$. Por ello, considero que una ley no debe permitir esta adulteración de documentos".

El labrado de una nueva acta de nacimiento con el sexo y nombre rectificado ${ }^{33}$ reproduce ficcionalmente la necesidad de una biografía -desde nacimiento a la adultez-coherente y lineal en términos de sexo-género. Nos parece interesante pensar esto en relación al hecho de que el acta de nacimiento "original" se labra sobre la definición médica del sexo genital "normal", lo cual finalmente también es una

\footnotetext{
${ }^{32}$ Durante la última dictadura cívico-militar argentina (1976-1983) se desarrolló no solo un plan sistemático de desaparición de militantes sociales, políticos y sindicales sino también de secuestro y apropiación de niños, generalmente hijos e hijas de las personas detenidas-desaparecidas. Según la organización Abuelas de Plaza de Mayo "Los niños robados como 'botín de guerra' fueron inscriptos como hijos propios por los miembros de las fuerzas de represión, dejados en cualquier lugar, vendidos o abandonados en institutos como seres sin nombre N.N. De esa manera los hicieron desaparecer al anular su identidad, privándolos de vivir con su legítima familia, de todos sus derechos y de su libertad" (http://www.abuelas.org.ar/).

${ }^{33}$ Según el artículo 6 de la Ley aprobada "Cumplidos los requisitos establecidos en los artículos $4^{\circ}$ y $5^{\circ}$, el/la oficial público procederá, sin necesidad de ningún trámite judicial o administrativo, a notificar de oficio la rectificación de sexo y cambio de nombre de pila al Registro Civil de la jurisdicción donde fue asentada el acta de nacimiento para que proceda a emitir una nueva partida de nacimiento ajustándola a dichos cambios, y a expedirle un nuevo documento nacional de identidad que refleje la rectificación registral del sexo y el nuevo nombre de pila. Se prohíbe cualquier referencia a la presente ley en la partida de nacimiento rectificada y en el documento nacional de identidad expedido en virtud de la misma".
} 
ficción. La Ley sancionada expresa ciertos pasajes de sentido: las intervenciones corporales dejan de ser entendidas como mutilaciones y pasan a ser cifradas como técnicas de reasignación sexual, así como los cambios registrales pasan de ser entendidos como una alteración de documentos público a una rectificación de datos.

Por otro lado, algunos legisladores expresaron la necesidad de regular el poder médico a través de las Leyes, reconociendo al mismo tiempo la dificultad de ello. Esto da cuenta, tal como se expresó previamente, de la autonomía relativa del campo médico. Según la Diputada Diana Conti (Alianza Frente Justicialista para la Victoria por la Provincia de Buenos Aires):

"La adecuación física, es decir, la intervención quirúrgica o cualquier otro procedimiento para modificar el cuerpo, es necesario plasmarla legalmente, por cuanto una intervención quirúrgica de cambio de sexo si no está autorizada penalmente es una lesión gravísima, porque en muchos casos anula una función primordial, como es la concepción o la procreación. Entonces, si no lo ponemos en una ley, los médicos van a hacer lo mismo que con el aborto no punible, es decir, que aunque hay aborto no punible no podemos lograr que los médicos procedan"34.

Según la Diputada Conti, la ausencia de una ley que regule el acceso a los tratamientos corporales por la población trans, dejaba espacio a la arbitrariedad y discrecionalidad médica tal como sucede actualmente en los casos de aborto no punible.

Por último, en torno a lo natural en relación a la noción de sexo observamos que legisladores y legisladoras se vieron ante la obligación de definir "qué es el sexo". De este modo, circularon dos planos: un sexo registral y un sexo biológico. Según el Diputado Tunessi:

\footnotetext{
${ }^{34} \mathrm{Al}$ momento de escritura del presente artículo, Argentina no cuenta con una ley que regule las prácticas de aborto. Según el Código Penal Nacional (Art. 85) el aborto constituye delito, no obstante, el mismo establece ciertas causales de abortos no punibles, es decir, legales (Art. 86). Sin embargo, como es de público conocimiento, muchos/as médicos/as actúan de modo discrecional frente a los casos que se adecuan a lo tipificado como aborto no punible, o sea, abortos legales, incumpliendo dicha reglamentación.
} 
"La identidad estática está conformada por el genoma humano, las huellas digitales, los signos distintivos; la identidad dinámica se refiere a los despliegues temporales influidos por la personalidad, construidos a partir de los atributos y características de cada persona, desde los éticos, religiosos y culturales, hasta los ideológicos, políticos y profesionales".

En este mismo sentido, el Diputado Ricardo Gil Lavedra (Partido Alianza Acuerdo Cívico y Social por la Ciudad Autónoma de Buenos Aires) expresó

"El sexo o el género no son acontecimientos naturales. Muchas veces las personas nacen con sexos determinados y no son percibidas como tal por ellas mismas. Esta categoría ha sido reconocida en el derecho como personas "trans" o "transgénero", es decir, no perciben la identidad del sexo que le viene naturalmente con lo que ellas sienten. En definitiva, lo que estamos consagrando es la posibilidad de dar prevalencia como un derecho inmanente a la personalidad el poder orientar su sexo de acuerdo a su autopercepción”.

Dicha caracterización implicó un planteo particular de la histórica tensión entre naturaleza-cultura en torno al sexo. Identificamos como emergentes de dicha tensión, la preocupación por la reversibilidad o irreversibilidad de los tratamientos (en particular los quirúrgicos). Ya sea que los legisladores abonaran una u otra idea, siempre remitían implícitamente a una idea de esencia original del sexo a la que se podría o no volver. La presunta imposibilidad de volver al sexo original $-\mathrm{y}$ en última instancia, verdadero- generó incomodidad y nerviosismo entre los y las legisladores/as. Según el Diputado Gustavo Ferrari (Partido Alianza Unión PRO por la Provincia de Buenos Aires):

"Evidentemente el cambio de sexo implica una decisión trascendental de cada persona, sobre todo cuando importa tratamientos quirúrgicos irreversibles. Por lo tanto, ella debe darse en un marco que asegure que sea la más adecuada para cumplir con el fin último del interesado y el fin último de la norma, es decir, su pleno y sano desarrollo social, psicofísico y sexual". 
Al adentrarse en la especificación de los aspectos estáticos y los aspectos dinámicos del sexo, este parecía quedar atrapado en una tensión dilemática: o bien es definido por las leyes de la naturaleza (y por ende inmodificable) o bien es fruto de la voluntad individual de las personas (imposible de ser problematizado). Para algunos legisladores el sexo estaría definido de una vez y para siempre por las leyes naturales o las leyes divinas. El diputado Alfredo Olmedo (Partido Alianza Somos Todos por la Provincia de Salta) afirmó

\begin{abstract}
“Cuando me incorporé a esta Cámara de Diputados juré por Dios y los Santos Evangelios. Lo recuerdo porque Dios creó la naturaleza y también al hombre y a la mujer (...) desde mi punto de vista no podemos ir en contra de la naturaleza. No me cabe en la cabeza que de acuerdo con esta propuesta cuando un niño tenga tres o cuatro años el padre le pueda preguntar a su hijo si quiere ser varón o mujer, si se quiere vestir de una u otra forma o si se quiere seguir llamando Pedro o de otra manera".
\end{abstract}

Quienes discutían con esta idea presentaban una idea construccionista del sexo, entendiéndolo como una construcción voluntariosa, individual y autónoma de los sujetos y que, por pertenecer al ámbito de la intimidad y la esfera de lo privado, debe ser resguardada y protegida por el Estado. En este sentido, el Diputado Felipe Solá (Partido Alianza Unión PRO por la Provincia de Buenos Aires) afirmó:

"El sexo depende mucho más de lo cultural, de lo simbólico y sobre todo de la voluntad de la persona que es dueña de su cuerpo en el sentido de saber cuál es su sexo (...) Es la voluntad que comienza a darse tempranamente la que determina el sexo; no es la cultura, ni siquiera es lo científico o la naturaleza. El hombre siempre ha dominado la naturaleza y su cuerpo. No podemos decidir cómo va a dominar su cuerpo él o ella. Lo decidirán ellos. Entonces, si alguien se llama José y quiere llamarse Josefa, tiene derecho a llamarse Josefa".

En este sentido, identificamos la presencia de un interrogante a lo largo del debate que no logra resolverse: ¿se modifica el sexo o 
solo su apariencia y/o su registro legal? ¿Hay sexo por fuera de la apariencia y el registro legal?

\section{REFLEXIONES FINALES E INTERROGANTES ABIERTOS}

Por las características ya comentadas de la Ley, la misma tiene propiedades que se destacan a nivel internacional en materia de derechos humanos: no establece ni como requisito ni como "aduana" el sometimiento a dispositivos psiquiátricos, jurídicos y/o médicos para acceder al cambio de sexo registral ni a los tratamientos quirúrgicos y hormonales. De hecho, estos tratamientos son presentados como un derecho al que se accede exclusivamente si la persona lo desea.

La Ley no es ni un espejo ni una determinación sobre lo social. Es un instrumento que se inserta en el marco de complejas relaciones. Para combatir la transfobia estructural es insuficiente, sin embargo plantea un piso institucional de derechos y disputa el sentido de lo legítimo, de lo sano y de lo normal en términos sexo/genéricos. Amplía los márgenes de la ciudadanía, pero la transfobia no puede ser eliminada por ley. Esta ampliación de los márgenes de la ciudadanía se llevó a cabo incorporando al ordenamiento binario de los géneros identidades y corporalidades que antes no cabían. Para ello fue necesario apoyarse en una idea de identidad autotransparente, voluntariosa e indivisa y en la ficción de un deseo unívoco en el marco de una historia biográfica coherente y lineal.

Cabe destacar que las voces autorizadas en el debate parlamentario, aparte de los/as legisladores/as, fueron en su mayoría activistas y militantes y en menor medida, representantes del poder judicial y ejectivo, quedando por fuera representantes del campo médico. No podríamos afirmar que dicha exclsuión fue lo que posibilitó la agilidad del proceso parlamentario que llevó a la aprobación de la ley. No obstante, que la letra de la ley se plasme en un servicio público, accesible y real para quienes lo necesiten requiere la implicación de una multiplicidad de actores sociales, especialmente de los/as efectores de salud. 


\section{POSTFACIO: LOS LÍMITES ESTRUCTUALES DE LA LEY}

Luego de la sanción de la Ley de Identidad de Género, los procesos de rectficación de nombre, sexo e imagen en los registros identificatorios oficiales fueron reglamentados mediante el Decreto $\mathrm{N}^{\circ}$ 1007/2012. De este modo, cualquier persona que desee modificar dichos datos, puede realizarlo sin ningun tipo de intermediario ante las oficinas del registro civil a lo largo de todo el país. Sin embargo, a dos años de su sanción, la reglamentación de la Ley en su aspecto sanitario aún se encuentra pendiente. Ello nos sugiere una serie de reflexiones.

Los/as actores/as que impulsaron el tratamiento y la sación de la Ley llevaron adelante una estretagia eficaz a fin de que reclamos sostenidos durante años fueran incorporados exitosamente a la agenda institucional y parlamentaria. Dicho proceso puede ser pensado en términos de la apertura de una ventana de oportunidades políticas abierta en un contexto favorable el cual supo ser leido por las organizaciones que actuaron en consecuencia. La no implicación de la comunidad médica en el proceso social que llevó a la sanción de la Ley hoy por hoy podría representar un obstáculo para la implementación de aquellos aspectos de la misma que requieren la intervención activa del cuerpo médico. Tal como se desarrolló previamente, la comunidad médica goza estructuralmente de una cierta autonomia relativa y llevó adelante históricamente una práctica tendiente a generar sus propias reglas y protocolos de acción. Más aún, frente a los procesos sociales sexo-genéricos han instituido prácticas patologizantes y normativizantes, sedimentadas luego culturalmente. A fin de revertir la tendencia histórica del campo médico es necesario un proceso complejo y más profundo, en otras palabras, una transformación social que disloque la heteronormatividad estructural y la violencia homotransfóbica. Si la estigmatización no se resuelve a un nivel cultural más profundo, corremos el riesgo de que la intolerancia hacia los cuerpos no adecuados a los términos sexo genéricos hegemónicos re-emerja como síntoma.

En este contexto nos preguntamos, ¿el campo médico puede constituirse como un saber técnico que cumpla con los deseos 
de transformación corporal de los sujetos, o es necesariamente un agente político que incide activamente en la subjetividad de las personas cuyos cuerpos interviene? ¿Cuál es el rol que debería cumplir el Estado para garantizar prácticas respetuosas de la autonomía de decisión de cada persona por parte del campo médico? ¿Puede el Estado despatologizar y desmedicalizar las identidades y los cuerpos?

\section{REFERENCÍAS}

ANDRÉU ABELA, J. Las técnicas del análisis de contenido: una revisión actualizada. In: Documentos de trabajo S2001/03. Sevilla: Centro de Estudios Andaluces, 1998. In: http://public. centrodeestudiosandaluces.es/pdfs/s200103.pdf, acceso el 29 de Junio de 2012.

BELMARTINO, . La atención médica en la Argentina del siglo XX. Buenos Aires: Siglo XXI, 2005.

- Nuevas reglas de juego para la atención médica en la Argentina: ¿quién será el árbitro? Buenos Aires: Lugar Editorial, 1999.

BENTO, B. A reinvenção do corpo: sexualidade e gênero na experiência transexual. Garamond: Rio de Janeiro, 2006.

BERKINS, L. y FERNÁNDEZ, J. La gesta del nombre propio: Informe sobre la situación de la comunidad travesti en la Argentina. Buenos Aires: Ed. Madres de Plaza de Mayo, 2005.

BERKINS, L. Cumbia, copeteo y lágrimas. Informe nacional sobre la situación de las travestis, transexuales y transgéneros, Buenos Aires: ALITT, 2007.

CABRAL, M. Ciudadanía (trans) sexual, 2003. In: www.ciudadaniasexual. org/publicaciones/Articulo_Mauro_Cabral.pdf, acceso el 16 de Julio 2012.

. Comparecer - un comentario, 2008. In: http://www.clam.org. br/uploads/conteudo/artigo_comparecer.pdf, acceso el 16 de Julio 2012.

La paradoja transgénero. In: Ciudadanía Sexual.org. Boletín Electrónico del Proyecto Sexualidades, Salud y Derechos 
Humanos en América Latina, n.18, Año 2, 2006. In: http://www. cnm.gov.ar/generarigualdad/attachments/article/280/La_paradoja_ transgenero.pdf, acceso el 16 de Julio 2012.

CONRAD, P. Sobre la medicalización de la anormalidad y el control social. In Psiquiatría Crítica. La política de la salud mental. Barcelona: Grijalbo, 1982.

. Medicalization and social control. Annual review of Sociology, Vol. 18, 1992, pp. 209- 232.

. The Medicalization of Society: On the Transformation of Human Conditions into Treatable Disorders. Baltimore: Johns Hopkins University Press, 2007.

FARJI NEER, A. Fronteras discursivas: travestismo, transexualidad y transgeneridad en los discursos del Estado argentino, desde los Edictos Policiales hasta la Ley de Identidad de Género. 2013. Tesis (Maestría en Investigación en Ciencias Sociales) - Facultad de Ciencias Sociales, Universidad de Buenos Aires, Argentina, 144 p.

FERNÁNDEZ, J. Cuerpos desobedientes. Travestismo e identidad de género, Buenos Aires: Edhasa, 2004.

FIGARI, C. Eróticas de la disidencia en América Latina. Brasil, siglos XVII al XX. Buenos Aires: CICCUS-CLACSO, 2009.

FOUCAULT, M. Seguridad, territorio, población. Buenos Aires: Fondo de Cultura Económica, 2006.

HILLER, R. Conyugalidad y ciudadanía: disputas en torno a la regulación estatal de las parejas gay lésbicas en la Argentina contemporánea. 2011. Tesis (Doctorado en Ciencias Sociales) Facultad de Ciencias Sociales, Universidad de Buenos Aires, Argentina, 254p

. Matrimonio igualitario y espacio público en Argentina. En Martín Aldao y Laura Clérico (Coords.). Matrimonio igualitario. Perspectivas sociales, políticas y jurídicas. Buenos Aires: Eudeba, 2010.

MENÉNDEZ, E. De sujetos, saberes y estructuras. Introducción al enfoque relacional en el estudio de la salud colectiva. Buenos Aires: Ed Lugar, 2009.

RAFFO, M.L.Ciudadanías en construcción. Un estudio sobre organizaciones de travestis en la Ciudad de Buenos Aires. Cuadernos Claspo, $\mathrm{n}^{\circ} .20$. Buenos Aires, CLASPO-Argentina, 2006. 
ROSE, N. Políticas de la vida: biomedicina, poder y subjetividad en el siglo XXI. La Plata: UNIPE, 2012.

SALESSI, J. Médicos maleantes y maricas. Higiene, criminología y homosexualidad en la construcción de la nación argentina (18711914). Rosario: Beatriz Viterbo Editora, 1995.

Recebido: 30/04/2014.

Aprovado: 02/12/2014. 